\title{
Micro Raman Spectroscopy: An Appropriate Method for the Characterization of Orientation and Crystallinity of Polypropylene
}

\author{
A. Gupper ${ }^{*}$, P. Wilhelm*, G. Kothleitner ${ }^{*}$, P. Zipper ${ }^{* *}$, D. Gregor-Svetec ${ }^{* * *}$, M. Gahleitner ${ }^{* * * *}$ \\ * Research Institute for Electron Microscopy, Graz University of Technology and Zentrum für \\ Elektronenmikroskopie Graz, Steyrergasse 17, A-8010 Graz, Austria \\ ** Institut für Chemie, Karl Franzens Universität Graz, Heinrichstraße 28, A-8010 Graz, Austria \\ ${ }^{* * *}$ Department of Textiles, University of Lubljana, Snezniska 5, SL-1000 Lubljana, Slovenia \\ ${ }^{* * * *}$ Borealis GesmbH, St. Peter Straße 25, A-4021 Linz, Austria
}

With an annual production of about 22 million tons polypropylene (PP) belongs to the most important synthetic materials. The wide range of applications and the great diversity of PP products concerning optical and mechanical properties demand a deep understanding of the materials properties. Fillers and additives are used for fine-tuning but the morphology is the main factor for controlling the final properties. In a semi-crystalline polymer it is controlled by the degree of chain orientation and crystallization. Furthermore the need of a fast and non-destructive characterization method for online analysis is growing and Raman spectroscopy seems to be an appropriate tool.

Raman spectroscopy is an excellent method for conformational analysis. Thus it is possible to determine the orientation of polymer chains within a sample. Even tacticity and amorphous contents can be identified using Raman spectroscopy. In our work we concentrated on polypropylene fibers. It was our goal to identify all Raman bands in the region between 800 and $1550 \Delta \mathrm{cm}^{-1}$, which are influenced by the orientation of the fiber with respect to the polarization of the incoming laser light. The other regions, mainly the one below $3000 \Delta \mathrm{cm}^{-1}$, were not considered because of strong peak overlapping and a more complicated evaluation of peak areas. To obtain the desired information a number of measurements under different sample orientation were performed. In this way we could obtain a rotational profile as shown in fig. 1, where only two wavenumber regions were selected. Spectra of a polypropylene fiber over the whole range of interest are shown in fig. 2, where orientation was changed by 90 degrees.

The other very important characteristic quantity in describing the sample morphology is the degree of crystallinity. Experiments in a hot/cold cell were performed and the peak intensities were observed over a wide temperature range. Disappearance of some peaks and a general broadening could be observed upon heating the sample. From the molten sample we could obtain data of nonoriented polypropylene. Under the assumption that orientation of chains can be neglected, when a melt is rapidly cooled from the liquid state to room temperature but under different shear conditions, we also registered spectra of semi-crystalline polypropylene with different degree of crystallinity but no orientation at all. This was very important in order to combine Raman information of orientation and crystallinity, which is necessary for investigations on injection molded samples, films or fibers as they are used in industry.

\section{References:}

[1] J. Karger-Kocsis, Polypropylen, An A-Z reference, Kluwer Academic Publishers, Dortrecht 1999

[2] M. Arruebarrena de Báez et al., Spectrochimica Acta Part A 51(1995) 2117 
[3] R.G. Snyder et al., Spectrochimica Acta Part A 20 (1964) 853

[4] G.Yu. Nikolaeva et al., Optics and Spectroscopy 85(3) (1998) 416

[5] D. Gregor-Svetec et al., Chem. Biochem. Eng. Q 9(3) (1995) 141

[6] Financial support for this work was granted by the "Forschungsförderungsfonds für die gewerbliche Wirtschaft" - project Nr.802914. The Raman instrumentation was financed in context of the „Impulsförderung für ACR-Institute“
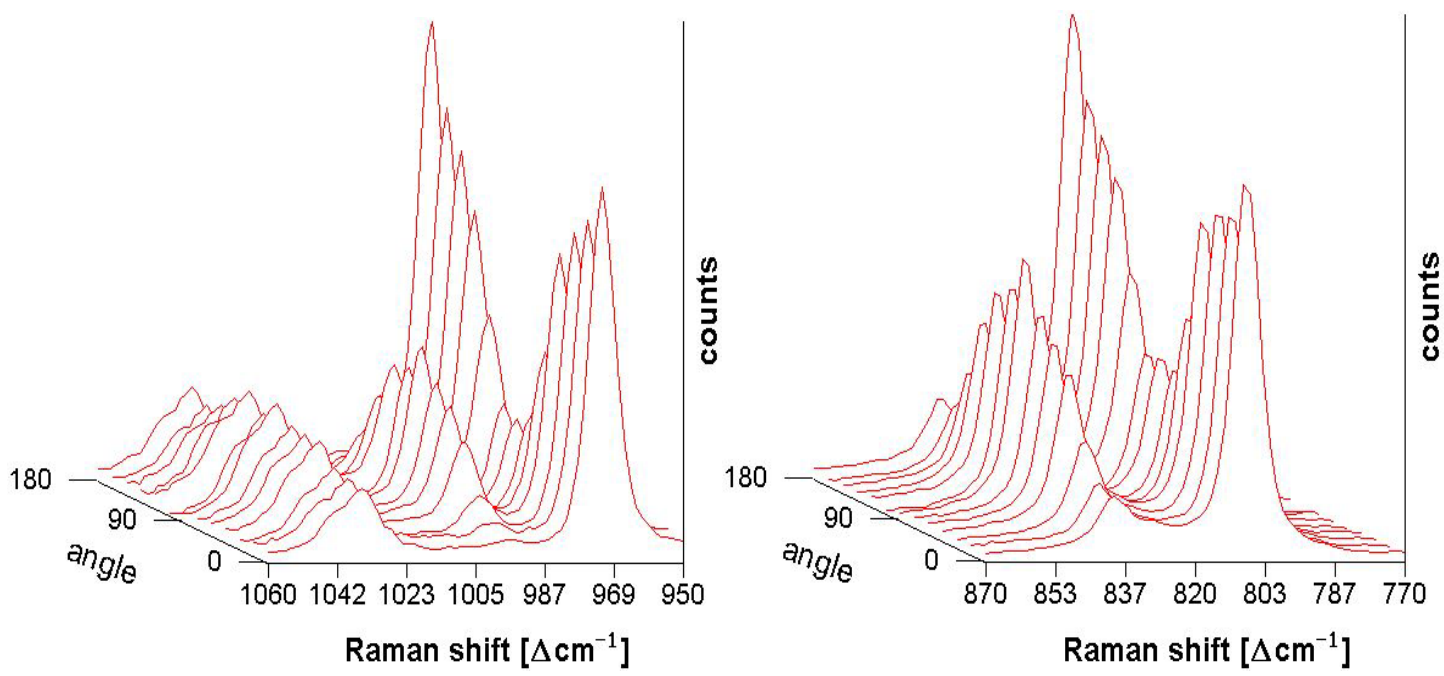

FIG. 1: Dependence of Raman band intensity from the angle between fiber and incoming laser polarization. The difference in angle between each spectrum is $15^{\circ}$.

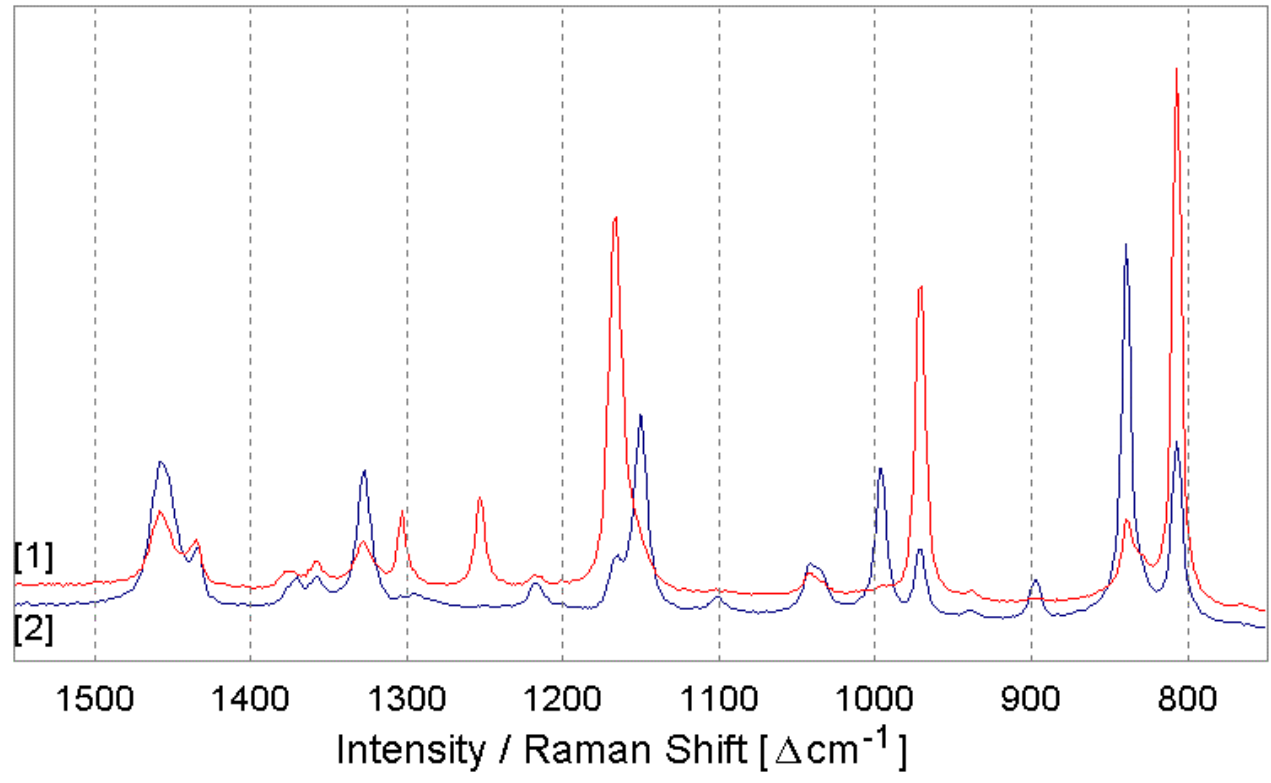

FIG. 2: Changes of band intensities in the Raman spectrum of polypropylene due to sample orientation. [1] Fiber is parallel and [2] perpendicular to incoming laser polarization. 